\title{
7 Eine Gefühlspolitik von affirmativen Gefühlskulturen
}

Das vorliegende Kapitel 7 dient der thematischen Einführung und wissenshistorischen Kontextualisierung des Untersuchungsgegenstands. Gefragt wird: Wie lässt sich eine Politik der ,Glückskulturen‘ wissenshistorisch erforschen?

\subsection{Emotionspolitisches Glückswissen: Eine Einführung}

In Anlehnung an Ute Freverts emotionshistorische Forschungen wird in der vorliegenden Untersuchung der Begriff Gefühlspolitik verwendet, um eine „Politik mit Gefühlen“ zu beschreiben. ${ }^{1}$ Im Rückgriff auf die Herrschaftstypologien des Soziologen Max Weber (1864-1920) argumentiert Frevert, dass ein bestimmendes Moment der Herrschaftslegitimation in der emotionalen Zustimmung der Bevölkerung liege. Diese Zustimmung, die sich in einer freiwilligen Fügsamkeit gegenüber einem neuen „Führer“ oder Herrscher manifestiere, sei, laut Weber, je nach historisch gewachsener Gesellschaftsform, auf der Grundlage einer Tradition, eines Gesetzes und/oder auf dem Charisma eines „Führers“ begründet. ${ }^{2}$ Die „gefühlsmäßige Hingabe“3 stelle sich nicht automatisch ein, sondern müsse, laut Frevert, mit Hilfe einer Gefühlspolitik erst geweckt werden: „eine in der Regel bewusste und reflektierte, mitunter generalstabsmäßig geplante Ansprache mit dem Ziel, Zustimmung zu erzeugen und affektiv zu modellieren.“4

Webers Überlegungen über die Bedingungen zur „Erhaltung des Charisma[s]“, welche eine Gefühlspolitik mitkonstituierten, sind für die Quellenanalysen in der vorliegenden Untersuchung erkenntnisreich. Der „Erhaltung des Charisma[s]“ läge Weber zufolge eine „Macht“ zugrunde, welche über das „persönliche Charisma“ eines „Führers“ hinaus ihre Wirkung „rational“ umforme: Weber nennt diese soziale Wirkungsmacht die „rationale Disziplin“5. Deren „spezifische Wir-

1 Vgl. Frevert 2012, 16. S. auch Einleitung, Kap. 1.4 und 2.1., 4.

$2 \mathrm{Zu}$ Webers Herrschaftstypologien s. auch Kershaws Forschung zum „Hitler-Mythos“, vgl. Kershaw 1999, Kershaw 2009. Vgl. auch Pyta 2010, Herbst 2010.

3 Zum Begriff vgl. Max Weber-Gesamtausgabe (MWG), I/23-6, 185f.; vgl. Rehberg 2014, 60.

4 Vgl. Frevert 2012, 21.

5 Zum Begriff: „Sie ist inhaltlich nichts anderes als die konsequent rationalisierte, d. h. planvoll eingeschulte, präzise, alle eigene Kritik bedingungslos zurückstellende, Ausführung des empfangenen Befehls, und die unablässige innere Eingestelltheit ausschließlich auf diesem Zwecke.“ Vgl. MWG I//22-4, 542-543, vgl. Weber 2009b, 163.

Ә OpenAccess. (c) 2021 Isabelle Haffter, publiziert von De Gruyter. (cc))BY-NC-ND Dieses Werk ist lizenziert unter einer Creative Commons Namensnennung - Nicht kommerziell - Keine Bearbeitung 4.0 International Lizenz. https://doi.org/10.1515/9783110661439-007 
kungen“ beruhten auf der „Qualität“ der „Disziplin“ als ein „Gemeinschaftshandeln eines Massengebilde[s]“. ${ }^{6}$ Was war damit gemeint? Nur aus „Gründen der Disziplin“ als „etwas in starkem Maße bewußt und rational Gewolltes“ halte die „ständisch[e] Lebensführung“ an „Stereotypisierung und Pflege“ fest, was wiederum „Rückwirkungen auf die gesamten, durch die jene Gemeinschaften irgendwie beeinflußten Kulturinhalte“ habe. ${ }^{7}$ Das gesellschaftsstiftende Merkmal einer Gefühlspolitik sei „die rationale Uniformierung des Gehorsams einer Vielheit von Menschen.“8 Im Kriegsfall weise die „rationale Disziplin“ folgende emotionspolitischen Eigenschaften auf:

„An Stelle der individuellen Heldenekstase, der Pietät, enthusiastischen Begeisterung und Hingabe an den Führer als Person und des Kultus der,Ehre' und der,Pflege‘ der persönlichen Leistungsfähigkeit als einer ,Kunst“ setzt sie die ,Abrichtung‘ zu einer durch ,Einübung“ mechanisierten Fertigkeit und, soweit sie an starke Motive, ethischen` Charakters überhaupt appelliert, ,Pflicht‘ und ,Gewissenhaftigkeit‘ voraus [...], alles im Dienst des rational berechneten Optimums von physischer und psychischer Stoßkraft der gleichmäßig abgerichteten Massen. Nicht daß der Enthusiasmus und die Rückhaltlosigkeit der Hingabe in ihr etwa keine Stätte hätten. Im Gegenteil: jede moderne Kriegsführung erwägt gerade die ,moralischen` Elemente der Leistungsfähigkeit der Truppe oft mehr als alles andere, arbeitet mit emotionalen Mitteln aller Art [...] und sucht in der Schlacht durch ,Eingebung، und noch mehr durch Erziehung zur ,Einfühlung، der Geführten in den Willen des Führenden zu wirken."9

Inwieweit der bei Weber wissenshistorisch kontextualisierbare Begriff einer explizit affirmativen Gefühlspolitik, die konstitutiv für eine rationale Disziplinierung eines menschlichen Kollektivs sei, Aufschluss für die quellenkritische Analyse der Fallbeispiele aus NS-Deutschland und der Schweiz gibt, wird im Verlauf der Untersuchung aufgezeigt.

Dass Gefühle und Politik eng miteinander verwoben sind, wusste bereits der Philosoph Aristoteles (384-322 v.u.Z.). Er legte in seinen Schriften zur griechischen Polis den Grundstein für eine politische Emotionstheorie, in welcher eine gefühlsgeleitete „Tätigkeit des Staatsmannes“ (politiké) eine „schädliche“ Regierungspolitik bedeute. ${ }^{10}$ In welchem wissenshistorischen Kontext stand Aristoteles’ Theorie über eine staatlich gelenkte Gefühlspolitik?

6 Vgl. Ebd.

7 Vgl. Ebd.

8 Vgl. Ebd.

9 MWG I/22-4, 543-545, vgl. Weber 2009b, 164.

10 Vgl. Aristoteles, Politik, I.5., 1254b, 1-11, Aristoteles 2014, 52. Für den Passagenhinweis vgl. Nielsen 2015, 32. Zu Aristoteles und Emotionen vgl. u. a. Fortenbaugh 1975. 
Seit der Antike bestand die Tendenz der „Verinnerlichung, Intellektualisierung und Moralisierung“ des Wissens über Gefühle und insbesondere über „Glück“.11 Die antiken Philosophen rückten „Glück“ in den „Bereich der menschlichen Machbarkeit“.12 Zur Beantwortung der Frage nach dem „guten“ (agathon) Leben fanden die philosophischen Schulen, von Demokrit bis Sokrates, von Platon bis Aristoteles, ganz unterschiedliche Antworten:

1. „Glück“ sei das höchste Ziel (telós), wonach jeder Mensch strebe, worauf er sein Leben ausrichte, um es zu erlangen,

2. nach dem Streben nach und Erlangen von „Glück“ fände der Mensch die wohlverdiente „Ruhe“,

3. „Glück“ sei aus verschiedenen „Gütern“ zusammengesetzt,

4. die Philosophie besäße sowohl in ihrer Methodik als auch in ihrer intellektuellen Tätigkeit als „beste Lebensform“ (aristós biós) eine Kulturtechnik „Glück“ herbeizuführen. ${ }^{13}$

Aufgrund ihres Glückswissens, ihrer Erkenntnisfähigkeit und ihres Urteilsvermögens wurde Philosophen noch lange bevor sie eine akademische Disziplin repräsentierten, die Autorität zugesprochen, als erste Glücksratgeber Anleitungen für eine „glückliche“ Lebensführung entwickeln zu können.

Einer der bis heute einflussreichsten moralphilosophischen Glücksratgeberautoren ist Aristoteles. In seinem Werk „Politik“ (griech. politiká) geht Aristoteles von der Annahme aus, dass der Mensch „von Natur aus ein politisches Lebewesen“ (zôon politikón) sei und folglich ein sozialpolitisches Leben führe. ${ }^{14}$ Diese Art der „Lebensform“ (biós) in einer „staatlichen Gemeinschaft“ (politiké koinonía) gehöre zur Naturgegebenheit des Menschen und sei ein „Glücksgut“. ${ }^{15}$ In der attischen Demokratie lebend, versuchte der griechische Philosoph, angesichts der zahlreichen autokratischen Herrschaftssysteme, eine Kernfrage des gemeinschaftlichen Zusammenlebens in der Antike zu beantworten, welche uns bis heute beschäftigt: In welchem Ausmaß ist es Aufgabe der Politik eines demokratischen, sozialistischen oder diktatorischen Staatsystems, die Gestaltung einer Gesellschaft auf eine glücksfördernde Weise in Anspruch zu nehmen? ${ }^{16}$ Aristoteles’ Antwort

11 Vgl. Horn 2011, 117.

12 Vgl. Glück in Gesellschaft. In: Henning 2011, 92-103, 97.

13 Vgl. Horn 2011, 118.

14 Aristoteles Politik, I.2., 1253a, 1-2. Vgl. u. a. Aristoteles 2014, 47.

15 „[S]taatliche Gemeinschaft“, Aristoteles Politik, I.2., 1252a, 7, vgl. Aristoteles 2014, 43. „Die Gemeinschaftlichkeit dieser Vorstellungen ruft aber eben das Haus und den Staat ins Leben“, Aristoteles Politik, I.2, 1253a, 18f, vgl. Aristoteles 2014, 47. Vgl. Henning 2011, 92.

16 Denkanstoß für diese Frage vgl. Henning 2011, 92. 
auf diese komplexe Frage wirkt zunächst einfach: „Glückseligkeit“ sei nur durch eine „Tätigkeit“ erfahrbar. Nur in einer tugendhaft ausgeübten „Tätigkeit“ läge „Glückseligkeit“. ${ }^{17}$ Um „Glückseligkeit“ zu erfahren sei es folglich sowohl im Interesse des Staates (und dessen „Verfassung“) als auch im Interesse jeder Einzelperson ein „tugendhafte[s]“ Leben zu führen:

„Daß nun also die beste Verfassung notwendig diejenige ist, deren Einrichtung dazu führt, daß es mit jedem Einzelnen aufs beste [sic] bestellt ist und ein jeder glückselig lebt, ist einleuchtend; strittig aber ist selber unter denen, die darin übereinstimmen, daß das tugendhafte Leben auch das wünschenswerteste [sic] ist, ob dabei ein den Staatsgeschäften (politikós) und der praktischen Tätigkeit (praktikós) gewidmetes Leben den Vorzug verdient [...].“18

Auch wenn sowohl Philosophen als auch Politiker der Meinung seien, so Aristoteles, dass ein „tugendhafte[s] Leben“ (aufgrund dessen Aussicht auf „Glück“) als „das wünschenswerteste [sic]“ gelte, sei man nicht darüber einig geworden, welche Lebensführung dieses beinhalte: seien es Staatsgeschäfte oder praktische Tätigkeiten. Um diesen Interessenskonflikt zu lösen, führt Aristoteles gewisse „äußere Güter“19 ins Feld, die im richtigen Maß sowohl für das Individuum als auch für den Staat ,glücksförderlich“ wirken sollten. Zu diesen „Güter[n] des Glücks“"20, welche „die beste Lebensform (aristos biós)“21 herbeiführen könnten, zählten eine politische Autonomie (Selbstregierung), in der sich ,alle“22 gleichermaßen, im Sinne einer sozialen Gleichheit, sowohl als Einzelperson als auch als Gemeinschaft entfalten und ausreichend Freizeit und Zugang zu Bildung für

17 „Glückseligkeit sei definiert als das rechte Handeln, das der Tugend entspringt [...]“, Aristoteles, Rhetorik, I., 1361a, vgl. Aristoteles 1999, 25.

18 Aristoteles Politik, VII.2., 1324b, 23-29, vgl. Aristoteles 2014, 296.

$19 \mathrm{Zu}$ den „äußeren Gütern“, welche die „Glückseligkeit“ positiv beeinflussen können, zählt A. „edle Abkunft, viele und gute Freunde, Reichtum, wohlgeratener und zahlreicher Nachwuchs, ein glückliches Altern, dazu noch körperliche Vorzüge wie Gesundheit, Schönheit, Kraft, Größe, sportliche Tüchtigkeit, Ansehen, Ruhm, Glück, Tugend [...]“. Vgl. Aristoteles Rhetorik, I., 1360b, vgl. Aristoteles 1999, 25. Diese Aspekte werden in den Kap. 8-14 zur Ratgeberliteratur wissenshistorische Kontinuität aufzeigen.

20 Aristoteles, Rhetorik, I., 1262a, vgl. Aristoteles 1999, 28.

21 „aristos biós“, Aristoteles, Politik, VII.1., 1323b, 16, vgl. Aristoteles 2014, 292.

22 Aristoteles, Politik, I.2., 1252a, 6, vgl. Aristoteles 2014, 43. Wobei z. B. „Sklaven“ nicht dieselben Rechte innerhalb der „staatlichen Gemeinschaft“ besaßen, wie athenische Männer, und folglich nach Aristoteles keine „Glückseligkeit“ erlangen könnten. Vgl. Aristoteles, Politik, III.9, 1280b, $3-8$. 
die ,höheren' Mußestunden genießen könnten (auf die Forderung nach der „besten Lebensform“ wird in den Kap. 8-14 weiterführend Bezug genommen). ${ }^{23}$

An erster Stelle stehe jedoch die Erziehung (paideía) zur Tugend (areté). Schließlich könne „Glückseligkeit“ als das gemeinsame Ziel (telós) des individuellen und politischen Lebens nur durch tugendhafte Handlung (eupragía) erreicht werden ${ }^{24}$ :

„Drum, wenn dies alles wahr und die Glückseligkeit (eudemonía) in die richtige Tätigkeit (eupragía) zu setzen ist, so wird sowohl für den Staat im ganzen [sic] als auch für den Einzelnen das beste Leben das tätige (praktikós) sein.“25

Durch die Aufklärung rückte „Glück“ (wieder) in den „Bereich menschlicher Machbarkeit.“26 Wie schon bei Aristoteles wurde „Glück“ zum Ziel und Zweck des gemeinschaftlichen Zusammenlebens und zur legitimen Forderung an die Politik. So war „The Pursuit of Happiness“ ein zentrales Anliegen der amerikanischen Unabhängigkeitserklärung (1776). ${ }^{27}$

Im Verlauf des 19. Jahrhunderts verschwand die Auseinandersetzung mit „Glück“ allmählich aus dem Interessensfeld der politischen Philosophie. Nicht nur Immanuel Kants und Karl Marx’ Glücksskepsis seien daran schuld gewesen, so Henning. ${ }^{28}$ Im 19. Jahrhundert des imperialen Nationalismus und liberalen Kapitalismus wurde „Glück“ erneut problematisiert und in den politischen Kontext uneingeschränkter Gewaltherrschaft gestellt. ${ }^{29}$ Politische Freiheit, soziale Gleichheit, egalitärer Bildungszugang und eine bestmögliche Entfaltung der Einzelperson in der Gesellschaft waren im 19. Jahrhundert hart umkämpfte Glücksgüter - und sind es bis heute geblieben. William Thompson verlangte 1824 sogar nach einer Wissenschaft, welche die „Principles of the Distribution of Wealth“ in Abhängigkeit zur „Human Happiness“ erarbeiten sollte. ${ }^{30}$ „Eugeniker“ wie Francis Galton (1822-1911) vertraten indes die Meinung, dass „das Glück“

23 „Das Glück ist, ebenso wie gewisse Fertigkeiten, Ursache einiger Güter, aber auch vieler außerhalb dieser Fertigkeiten [...]“, vgl. Aristoteles, Rhetorik, I., 1362a, vgl. Aristoteles 1999, 28.

24 Vgl. Aristoteles Politik, VII.2, 1325a, 7-11; Aristoteles Politik, VII.2, 1325b, $14-17$.

25 Aristoteles Politik, VII.3, 1325b, 14-17, vgl. Aristoteles 2014, 301.

26 Vgl. Henning 2011, 97.

27 Mehr zum Verhältnis von Wohlstaat und „Glück“ vgl. Nielsen 2015, 33.

28 Vgl. Henning 2011, $97 \mathrm{f}$.

29 Vgl. ebd., $98 \mathrm{f}$.

30 Werk von 1824: „An Inquiry into the Principles of the Distribution of Wealth most conductive to Human Happiness“, vgl. Thompson 1968. Hinweis auf das Werk vgl. Henning 2011, 100. 
einer Gesellschaft, ohne Rücksicht auf Einzelpersonen, biologistisch ,erzeugbar sei. $^{31}$

Mit Blick auf den Untersuchungszeitraum 1933-1945 stellt sich die Frage, welchen wissenshistorischen Wandel Aristoteles' Glückswissen über eine gelenkte Gefühlspolitik und eine durch Erziehung eingeübte „tugendhafte Tätigkeit“ zum Erlangen von „Glückseligkeit“ (Aristoteles) erfuhr.

\subsection{Der Januskopf einer affirmativen Gefühlspolitik}

Die Nationalsozialisten setzten nach dem Vorbild 1. „religiöser Praktiken, Rituale und Semantiken“, 2. der Machtregime der Frühen Neuzeit und 3. der sozialdemokratischen Massenkundgebungen während der Weimarer Republik auf eine Politik der Gefühle. ${ }^{32}$

Ausgehend vom aktuellen Forschungsstand wird in der vorliegenden Untersuchung gefragt:

1. Welches historische Glückswissen über eine moralische Lebensführung prägte die Gefühlspolitik im Nationalsozialismus?

2. Können Wandel, Kontinuitäten und Ambivalenzen einer affirmativen Gefühlspolitik im transnationalen Vergleich zwischen NS-Deutschland und der Schweiz festgestellt werden?

Zur Beeinflussung der Massen im NS-Regime wurde eine affirmative Gefühlspolitik eingesetzt. Dieses wirkungsmächtige Propagandainstrument hatte einen Januskopf: Die Kehrseite der Politik einer ,Glückskultur‘ war ihre Unberechenbarkeit und Schnelllebigkeit, welche machthabende NS-Funktionäre zwang, kontinuierlich sowohl politische wie auch wirtschaftliche Erfolge aufzuweisen. ${ }^{33}$

31 Vgl. Henning 2011, 99. Auf scheinbar biologistisch erzeugbares bzw. „rassenhygienisches“ „Glück“ wird im Kap. 14 nochmals Bezug genommen.

32 Vgl. Frevert 2015, 482.

33 Ian Kershaw analysiert im Rückgriff auf Max Webers charismatischen Herrschaftstypus die „Image-Bildung“ und „Image-Rezeption“ des „Hitler-Mythos“. Kershaw betont die Fragilität des „Führerkults“, dessen Beliebtheit vom subjektiven Urteil seiner Anhänger`innen abhängt und der sich daher gezwungen sieht, kontinuierlich Erfolge aufzuweisen, um seine Herrschaftslegitimation zu sichern. Vgl. Kershaw 1999, $21 \mathrm{ff}$. Nach Webers Ansicht muss sich der charismatische „Führer“ die „Anerkennung“ seiner Anhänger^innen durch Bewährung in Form von „Wunder[n], Erfolge[n], Wohlergehen der Gefolgschaft oder der Untertanen“ erlangen. „Nur so lange gilt er dafür [als charismatischer Herr], als er das kann. Ist ihm Erfolg versagt, so wankt seine Herrschaft." Weber 2009c, 222f. Weber bezieht sich in diesem Beispiel u. a. auf Napoleon, Jesus und Perikles. 
Vor dem Hintergrund der 1929 einsetzenden Weltwirtschaftskrise, die eine hohe Arbeitslosigkeit mit sich brachte, waren die Anzeichen einer wirtschaftlichen Konjunktur im Verlauf der 1930er Jahre ein Garant für eine mehrheitlich positive Stimmung in NS-Deutschland. ${ }^{34}$ Die ökonomischen Erfolge und sozialen Verbesserungen wusste das NS-Regime als Errungenschaft einer neuen Wirtschaftskraft und Sozialpolitik propagandistisch auszuschlachten. Die nach Kriegsausbruch in der Bevölkerung ausgelöste Kriegseuphorie, einhergehend mit einer Bewunderung für Hitlers erfolgreiche „Blitzkriegsstrategie“, wurde allerdings bereits im Winter 1940/41 von einer zunehmenden Skepsis gegenüber einem raschen Kriegsausgang abgelöst. Persönliche und wirtschaftliche Sorgen der Bevölkerung während des Kriegsalltags (Rationierungen, Lebensmittelengpässe, Fliegerangriffe, ansteigende Kriegsopferzahlen) waren Gründe, warum sich die Stimmung spätestens seit Frühjahr 1942 zunehmend zu verschlechtern begann. ${ }^{35}$

Für die Schweiz sind ähnliche emotionale Schwankungen in Abhängigkeit mit politischen und wirtschaftlichen Erfolgen beziehungsweise militärischen Bedrohungs- und Krisenszenarien zu beobachten: Nach den ersten Schockjahren der Wirtschaftskrise von 1929 erholte sich die Schweizer Wirtschaft in den 1930er Jahren kontinuierlich bis zum Kriegsausbruch im Herbst 1939. ${ }^{36}$ Im Kontext der zunehmenden faschistischen und nationalsozialistischen Bedrohung durch die Nachbarländer Italien und Deutschland (seit 1938 auch Österreich, bzw. „Ostmark“, seit 1940 „Vichy-Frankreich“) wurde die „geistige Landesverteidigung“ Ende der 1930er Jahre als staatlich verordnetes Propagandainstrument eingesetzt, um sich gegen die ideologischen Einflüsse des Faschismus und Nationalsozialismus zur Wehr zu setzen. Auf emotionspolitischer Ebene war die „geistige Landesverteidigung“ ein Instrument einer nationalen Abgrenzungs- und zugleich emotionspolitischen Distinktionsstrategie, um in der Bevölkerung affirmative Gefühle gegenüber den Schweizer Werten (Freiheit bzw. nationale Unabhängigkeit, „nationale Selbstbesinnung“‘37, Demokratie, Föderalismus, Neutralität) und ihrer Kultur zu wecken. ${ }^{38}$

34 Vgl. Der Staatsmann Hitler: Die Phase der nationalen Hitler-Euphorie. In: Kershaw 1999, 151164.

35 Vgl. Frühe Siege. In: Wildt 2008, 145-157. Vgl. Der Verfall des Hitler Mythos. In: Kershaw, 83274, 199.

36 Vgl. Tanner 2015.

37 Vgl. Bonjour 1944. In Edgar Bonjours (1898-1991) Gedenkrede zur 500-Jahr-Feier der „Schlacht bei St. Jakob an der Birs“ am 26. August 1944 in Basel werden aus Sicht des Geschichtsprofessors die historischen und zeitgenössischen Wesenszüge des Schweizerischen Selbstverständnisses benannt. Bonjour war von 1935-1968 ord. Professor für Schweizergeschichte und Neuere Allgemeine Geschichte an der Universität Basel. Zu seiner Person vgl. Kreis 2004. Mehr zum Schweizer Selbstverständnis 1933-1945 vgl. Tanner 2015, s. Kap. 15. 
Die „geistige Landesverteidigung“, so die These der vorliegenden Untersuchung, weist insbesondere in ihrer kulturnationalistischen Programmatik Züge einer affirmativen Gefühlspolitik einer schweizerischen ,Glückskultur' auf. ${ }^{39}$ Dahinter stand die Absicht, nicht nur ein gemeinschaftsstiftendes, sondern auch ein leistungsorientiertes und autosuggestives Zusammengehörigkeits- und Solidaritätsgefühl zu erwecken, auf das sich im Kriegsfall zurückgreifen ließe. Im Sinne einer landesweiten Integrationspolitik verfolgten die Verfasser der sogenannten „Bundesrätlichen Kulturbotschaft“40 das Ziel, sich auf überregionale, übersprachliche und vornehmlich christliche (!) ${ }^{41}$ Konfessionsgrenzen übergreifende Kulturwerte der Schweiz zu verständigen, um eine überparteiliche Konsensfindung zu erwirken. Folglich sollte die „geistige Landesverteidigung“ eine nationalpolitische Integrationsmöglichkeit für alle Gesellschaftsschichten bieten, um alte Klassenkämpfe $\mathrm{zu}$ beschwichtigen. ${ }^{42}$

Am 9. Dezember 1938 wurde die sogenannte „Kulturbotschaft“, federführend von Bundesrat Philipp Etter (1891-1977), programmatisch verkündet. ${ }^{43}$ Dieser Zeitpunkt war nicht zufällig gewählt: Nachdem Hitler bereits im März 1936 mit der militärischen Wiederbesetzung des Rheinlandes seine expansiven Machtansprüche mittels diplomatischer Schachzüge geltend gemacht hatte, erwirkte der Diktator unter der Parole „Heim ins Reich“ im März 1938 abermals ohne Waffengewalt den „Anschluss“ der „Ostmark“ (Österreich). Im Spätsommer 1938 gelang Hitler die Besetzung des tschechischen „Sudetengebiets“ und er ließ seine Annexion im „Münchner Abkommen“ vom 30. September 1938 international für rechtsgültig erklären. Bis zum Angriff auf Polen am 1. September 1939 gelang Hitler nach seinem völkerrechtswidrigen Einmarsch in Prag auch noch am

38 Zur Identitätskonstruktion der Schweiz als Nation vgl. Marchal und Mattioli 1992.

39 Mehr dazu s. Kap. 15, 16.

40 Vgl. Botschaft des Bundesrats 1938.

41 Zum Antisemitismus in der Schweiz 1900 -1945 vgl. Kreis 2008, Kamis-Müller 2000, Altermatt 1999, Kreis u. a. 1998, Mattioli 1998.

42 Vgl. Jorio 2006. Zur Schweiz im Zweiten Weltkrieg vgl. Kreis 2011, insbesondere der sog. „Bergier-Bericht“, der Schlussbericht der „Unabhängigen Expertenkommission Schweiz-Zweiter Weltkrieg“, vgl. Unabhängige Expertenkommission 2002 sowie deren Forschungsbände 1-26. Für die vorliegende Arbeit insbesondere der 17. Band zur Schweizer Flüchtlingspolitik, vgl. Unabhängige Expertenkommission 2001. Zur Flüchtlingspolitik im Kontext von Theater und Literatur vgl. Amrein 2013, Schulz 2012, Amrein 2004. S. auch Einleitung, Kap. 2.4.

43 Vgl. u.a. Sarasin 2014. Philipp Etter, SKVP-Politiker (heute: CVP) von 1934 bis 1959 im Bundesrat, Eidgenössisches Departement des Innern, federführend bei der Entwicklung der „geistigen Landesverteidigung“. Seine Haltung: konservativ-katholisch, antisemitisch, von seiner innerschweizerischen Heimat geprägt, vertrat er das Konzept eines christlichen, ständestaatlichen und autoritären Staates. Mehr zur Person vgl. Widmer 2017. 
15. März 1939 die Angliederung des litauischen Memelgebiets ins „Großdeutsche Reich“. 44

Die militärische Bedrohung, die von NS-Deutschland ausging, musste emotionspolitisch in der Schweizer Bevölkerung in eine möglichst hoffnungsfrohe und zuversichtliche Grundstimmung umgelenkt werden. Eine allgemeine Panik aus Angst, das nächste Annexionsopfer $\mathrm{zu}$ werden, sollte vermieden werden. Auch in der Schweiz zeigte sich die Kehrseite der affirmativen Gefühlspolitik in ihrer schwer zu kanalisierenden Wirkungsmacht: Neben positiven, konnte sie gerade in rechts-konservativen und nationalistischen Kreisen, unter Nazisympathisant*innen und im anti-demokratischen Umfeld der rechtspopulistischen „Nationalen Front“, auch negative Gefühle gegenüber dem demokratischen Staat und deren Politikern hervorrufen (darauf wird in den Kap. 15 und 16 Bezug genommen). ${ }^{45}$

Nachdem im Rückgriff auf den Begriff der affirmativen Gefühlspolitik die Ambivalenz einer Politik der ,Glückskulturen' wissenshistorisch kontextualisiert wurde, wird im Folgenden in wissenshistorischem Bezug zur NS-Ideologie und deren Entwurf einer nationalsozialistischen Lebensführung der Wandel lexikalischen Glückswissens aufgezeigt.

\subsection{Glückswissen und Lebensführung. Ein lexikalischer Bedeutungswandel}

Die nationalsozialistische Ideologie, in welcher die Einzelperson gegenüber dem „Volkskörper“ keinen Wert besaß, war auf „Rasse“ und „Blut“ begründet. Alles Handeln, das zum Erhalt der „arischen Rasse“ diente, war unter der nationalsozialistischen Gesetzgebung gerechtfertigt. Im Umkehrschluss hatte die nationalsozialistische „Rassenideologie“, auf der die NS-Moral beruhte, zur Folge, dass jede Kategorie von „Artfremdem“ oder „Nichtarischem“ potentiell die Lebensführung der „arischen Rasse“ gefährde und daher ohne Rücksicht auf humanistische oder religiöse Ethik gewissenlos vernichtet werden sollte. ${ }^{46}$

Der „Volksgenosse“ sollte, seit der Errichtung des NS-Regimes im Frühjahr 1933, nicht länger in einem individuellen Glücksempfinden aufgehen, wie es der Utilitarismus Jeremy Benthams („Das größte Glück der größten Zahl“) vorsah.

44 Vgl. Der Weg in den Krieg. In: Wildt 2008, 134-145.

$45 \mathrm{Zu}$ rechtsintellektuellen Kreisen in der Schweiz vgl. Gonzague de Reynold. Vordenker, Propagandist und gescheiterter Chef der „Nationalen Revolution“. In: Mattioli 1995, 13-156.

46 Zur nationalsozialistischen Rasse und Moral vgl. Bialas 2014, 19-62 und 63-110. 
Neben dem individuellen Streben nach „Glück“ wurde der epikureische „Eudämonismus“ als eine „Befriedigung egoistischer Triebe und Lüste [...], ohne Verantwortungsbewusstsein“ gegenüber der „Volksgemeinschaft“, abgetan und im Sinne der nationalsozialistischen Moralvorstellung 1938 in Meyers Lexikoneintrag über „Glück“ als „unsittlich“ bezeichnet. ${ }^{47}$ Dieser wissenshistorische Bedeutungswandel nach 1933, der von Kontinuitäten und Ambivalenzen geprägt ist, lässt sich anhand des lexikalischen Glückswissens in deutschsprachigen Sprachwörterbüchern und Enzyklopädien untersuchen, wie die folgende Analyse exemplarisch aufzeigen wird.

Warum eignet sich das Medium Lexikon besonders für eine wissenshistorische Untersuchung? Worin liegt der Erkenntnisgewinn? Die Untersuchung geht von der Annahme aus, dass Gefühlswissen in wissenschaftlichen, politischen und kulturellen Gesellschaftsbereichen transnational zirkulierte und wechselseitig (re-)produziert wurde. Für eine wissenshistorische Untersuchung ist es daher entscheidend, jene Orte und Objekte ausfindig $\mathrm{zu}$ machen, mittels derer (Re-) produktionen von und Reflexionen über Glückswissen transnational angestellt wurden. Darüber hinaus gilt es, in einem historischen Vergleich des lexikalischen Glückswissens aus der Zeit vor und nach 1933 aufzuzeigen, welchen wissenshistorischen Wandel es durchlief und welche Brüche, Kontinuitäten und Ambivalenzen dabei zutage traten.

Sprachlexika entstanden im 18. Jahrhundert im Kontext bürgerlicher Emanzipationsprozesse und damit verknüpfter sozialpädagogischer Konzepte einer nationalen Volkserziehung. Als aufklärerisches Bildungsmedium konzipiert, stellt das Lexikon eine wertvolle Quelle dar, welche über das zu einem bestimmten Zeitpunkt festgelegte Wissen Auskunft gibt. ${ }^{48}$ Das Lexikon, ein gesellschaftlicher Informationsproduzent, -transformator und -speicher zugleich, kann aufgrund seiner hohen Auflagenzahl und seiner hegemonial normierend wirkenden Wissensvermittlung als ein Diskursmedium von transnationalem Gefühlswissen gelten. Dennoch muss das Lexikon, wie jede andere Textquelle, historisch-kritisch hinterfragt werden. Gerade in Bezug auf die Rezeptionsseite darf bezweifelt werden, dass die beabsichtige soziale Reichweite dieses Mediums in der Bevölkerung breit wirkte. ${ }^{49}$ Dennoch ist das Lexikon gerade vor dem Hintergrund der propagandistischen Zensurmechanismen in NS-Deutschland eine aufschlussreiche Quelle, über welche sich die hegemonial geführte Zirkulation nationalsozialistischen Glückswissens erschließen lässt.

47 Art. Glück 1938.

48 Vgl. Haltern 1976, 62.

49 Vgl. Frevert 2011, 16f. 
Im Folgenden soll der wissenshistorische Wandel von Glückswissen anhand von Brockhaus-Editionen, von Spamers Konversations-Lexikon und von Meyers Lexikon aufgezeigt und wissenshistorisch kontextualisiert sowie quellenkritisch analysiert werden.

$\mathrm{Zu}$ Beginn ihres Lexikoneintrags über „Glück“ in Meyers Lexikon von 1938 arbeitet die Lexikonautor`in wesentliche Merkmale lexikalischen Glückswissens heraus:

„Glück /(Glückseligkeit), Empfindung des Zustandes der Erfüllung eines Wunsches, Zieles oder einer Aufgabe, urspr. sow. gelingen (,es glücke ihm“). Je nach der Haltung des einzelnen oder der weltanschaulichen Bestimmung einer Zeit sind Sinn und Inhalt des G. zw. den Extremen in der menschl. Entwicklung gespannt.[...]“50

Brüche, Kontinuitäten und Ambivalenzen kennzeichnen, der NS-Lexikonautor`in zufolge, Glückswissen. Es ließe sich einerseits entsprechend der individuellen, moralischen Haltung“ eines Menschen, andererseits aufgrund einer Staatsräson oder Staatsideologie sowie, je nach dynamisch sich wandelndem historischen Kontext, „zw. den Extremen in der menschl. Entwicklung“ anders definieren. Weiter heißt es im Lexikoneintrag:

„[...] Dem materialistisch bestimmten Eudämonismus, der in der Befriedigung egoistischer Triebe und Lüste sein G. empfindet ohne Verantwortungsbewusstsein gegenüber den eigentlichen menschl. Aufgaben, steht der ethische Rigorismus gegenüber, der das G.streben als unsittlich verwirft. Das mit dem G. meist verbundene Aufgehen in Passivität und Gefühl hat von jeher das Misstrauen kämpferischer Menschen gegen die Preisung des G. (,Das grösstmögliche G. der grösstmöglichen Zahl!‘, wie es z. B. J. Bentham forderte) wachgerufen: Kant kämpfte für die Pflicht gegen das G.; Nietzsche erklärte: ,Trachte ich denn nach meinem G.? Ich trachte nach meinem Werke'. Der nordische Mensch sucht daher in seiner Lebensauffassung nicht das wunschlose Glücklichsein, sondern die Freude, um neue Kraft zu seinem Werk zu finden." ${ }^{\text {51 }}$

In wissenshistorischer Kontinuität zum Dichotomie-Diskurs über „Gefühl“ vs. „Verstand“, wie er exemplarisch bei René Descartes (1596-1650) geführt wurde, deutet die Lexikonautor*in das Glückswissen 1938 NS-ideologisch um: Einem „kämpferische[n] Mensch[en]“, wie es das rassistische Ideal eines „nordischen Menschen“ verkörpere, seien „Passivität und Gefühl“ suspekt, folglich auch „Glück“. Den Begriff „Gefühl“ kennzeichnet die Autor`in mit der moralischen Wertung der „Passivität“, den „Verstand“ hingegen mit dem positiv konnotierten

50 Art. Glück 1938.

51 Ebd. 
Attribut der „Aktivität“. In Wissenskontinuität zum Moralphilosophen Kant interpretiert die Lexikonautor*in die moralische „Pflicht“ und „menschl. Aufgabe“ eines „nordischen Menschen“ „gegen das G.“ und spricht sich in ihrer ambivalenten Kant-Rezeption gegen „Passivität“ und für einen ethisch rigorosen Aktionismus aus. Ähnlich habe auch Friedrich Nietzsche (1844-1900) argumentiert, wenn dieser behauptete, dass der Mensch nach „dem Werke“ trachte, so die Autor^in (darauf wird später im Kapitel nochmals Bezug genommen). Gemäß dem Lexikoneintrag lag das Ziel der nationalsozialistischen Lebensführung nicht im „wunschlose[n] Glücklichsein“, sondern in der „Freude, um neue Kraft zu seinem Werk zu finden. ${ }^{\text {"52 }}$ Entsprechend lautete die nationalsozialistische Arbeitsmoral „Kraft durch Freude“, so die These der vorliegenden Untersuchung.

Mit Blick auf die Frage nach einer Wissenszirkulation von lexikalischem Glückswissen dient die im englischen Exil entstandene Aufzeichnung des antifaschistischen Publizisten Sebastian Haffner (1907-1999) exemplarisch für eine wissenshistorische Vergleichsanalyse. Haffner äußerte sich 1939, ein Jahr nach der Publikation des Lexikoneintrags, wie folgt über das „Glück“ des „Durchschnittsdeutsche[n]":

\begin{abstract}
„Ich erwähnte schon [...], dass die Begabung meines Volkes zum persönlichen Leben und persönlichen Glück ohnehin schwächer ausgebildet ist als die anderer Völker. Ich habe später in Frankreich und England [...] nicht ohne Neid beobachtet und nachempfinden gelernt, welche Fülle von unverwelklichem Glück [...] etwa der Franzose aus dem verständiggeistreichen Essen und Trinken, dem männlichen Redestreit und der heidnisch-künstlerisch kultivierten Liebe, der Engländer aus seinen Gärten, dem Umgang mit Tieren und seinen vielen kindlich-ernsthaft betriebenen Spielen und Hobbys gewinnt. Der Durchschnittsdeutsche hat nichts Entsprechendes. Nur eine bestimmte Bildungsschicht [...] findet ähnliche Lebensinhalte und Lebensfreuden in Büchern und Musik, eigenem Denken und dem Bilden einer eigenen ,Weltanschauung'. [...] treu und etwas sentimental bewahrte und gepflegte wenige Freundschaften, schließlich, nicht zu vergessen, ein inniges und intensives Familienleben - das sind die Lebensgüter- und freuden, die in dieser Schicht zu Hause waren." ${ }^{53}$
\end{abstract}

Haffners Glückswissen ist nicht mit dem lexikalischen Glückswissen in Meyers Lexikon kompatibel. Dennoch verweist Haffner auf eine, gemäß der literaturwissenschaftlichen Glücksforschung, typisch „deutsche“54 Charakterisierung des „Glücks in der Bescheidung““55. Bereits Ende des 19. Jahrhunderts trat dieses Ge-

52 Vgl. ebd.

53 Haffner 2000, 71.

54 Vgl. Brenner 2002, 251.

55 Tanzer 2011, 53. 
fühlswissen als „Darstellung des Vollglücks in der Beschränkung“56, wie in Jean Pauls Erzählung „Leben des vergnügten Schulmeisterlein Maria Wutz in Auenthal. Eine Art Idylle“ (1793) oder in Dramen wie Hermann Sudermanns „Glück im Winkel“ (1896), ambivalent in Erscheinung. 1922 vermerkte der Brockhaus. Handbuch des Wissens in vier Bänden dieses spezifische Gefühlswissen als eine Folge der wechselseitigen Wissenszirkulation zwischen Wissenschaft und Praxis in einem separaten Eintrag. ${ }^{57}$

Verallgemeinerungen, wie die Annahme einer deutschen bildungsbürgerlichen Glücksskepsis als Folge einer vernunftorientierten Aufklärung oder die des kleinen privaten „Glücks“ eines „Durchschnittsdeutschen“, greifen unter der Prämisse von sich wandelnden und ambivalenten Wissenskulturen zu kurz. Spamers Illustrirtes Konversations-Lexikon wies bereits 1893 auf die Möglichkeit eines individualisierten Glückskonzepts hin:

„Glück ist zunächst derjenige Seelenzustand, in welchem wir uns zufrieden, d.h. unser Dasein in Übereinstimmung mit unserem Zwecke finden. Je nachdem nun der Zweck des Daseins in den Besitz geistiger oder sittlicher oder materieller Güter gesetzt wird, ist diese Glücksempfindung oder Glückseligkeit bei verschiedenen Menschen verschieden.“58

Die Vorstellung eines persönlichen „Glücks“ „im Besitz geistiger“, „,sittlicher“ und „materieller“ Güter, wie sie an der Schwelle zum 20. Jahrhundert in Spamers Lexikon formuliert (und bereits von Aristoteles beschrieben wurde), sollte kennzeichnend für das individuelle Glücksstreben der Moderne werden. ${ }^{59}$

1926 stellte Meyers Lexikon-Ausgabe das „Subjekt“ ins Zentrum der Begriffsbestimmung:

„Glück, im objektiven Sinn eine Lebenslage, im subjektiven ein Gemütszustand. Im erstern [sic] Sinne bezeichnet es den Besitz eines an sich wünschenswerten Gutes (Gesundheit, Reichtum usw.), dessen Erlangung weder gewiß [sic], noch auch nur (für den Betreffenden) bes. wahrscheinlich war; im letztern [sic] Sinne das aus jenem Besitz entspringende Lustgefühl, dann überhaupt den Zustand vollkommener innerer Befriedigung (Glückseligkeit ${ }^{60}$, s.d.). “61

56 Paul 1963, 258. Vgl. Tanzer 2011, 53.

57 Art. „Glück im Winkel, Das, sprichwörtlich gewordener Titel eines Schauspiels von Hermann Sudermann (1896)“ Vgl. Art. Glück im Winkel 1922. Diese Ausgabe enthält keinen Einzeleintrag zu „Glück“.

58 Art. Glück 1893.

59 Zur individualisierten Glückssuche in der Moderne vgl. u. a. Duttweiler 2007, Kleiner 2014, Eitler 2015.

60 „Glückseligkeit (griech. Eudämonie), der Zustand des sinnlich-vernünftigen Wesens, in dem es nicht nur seine Bedürfnisse, sondern auch seine Wünsche befriedigt sieht, oder (nach Kant), 
Mit einer zunehmenden, populärwissenschaftlichen Einflussnahme der Mediziner^innen, Neurolog^innen, Psycholog^innen und Psychiater^innen verfestigte sich seit 1900 die naturwissenschaftliche Lehrmeinung, dass sich Gefühle nicht länger als „eigenständige Kraft“ im Dreieck zwischen Körper - Seele - Geist verorten ließen, sondern Teil des Körpers seien. Mit dieser „Somatisierung der Gefühle“ ging auch eine „Psychiatrisierung der Gefühle“ einher, ${ }^{62}$ deren Einfluss das Vokabular des Lexikonartikels abbildet: Der Eintrag legt nahe, zwischen einer aus der Außenperspektive zu beurteilenden „Lebenslage“ und einer psychologischen Innenperspektive, vom Standpunkt des „Gemütszustands“ eines Individuums aus, zu unterscheiden. Auffällig ist, dass auf eine moralische Wertung zwischen dem „Besitz an sich wünschenswerte[m] Gute[m]“ und dem „aus jenem Besitz entspringende[n] Lustgefühl“ verzichtet wurde. Im Vergleich dazu wird in dieser Lexikondefinition jedoch expliziter als in den bisherigen Artikeln auf die (Un-)Wahrscheinlichkeit eines Glückserlangens und auf den Faktor Zeit eingegangen. So verweist die unbekannte Autor`in auf das Streben nach einem „Glück“, dessen Erreichen womöglich nie realisiert werden kann. Die Ungewissheit und Unsicherheit über die Realisierbarkeit der persönlichen Wünsche ist kennzeichnend für das zeittypische Konzept der „Krise“ des selbstbestimmten Individuums in der Moderne. ${ }^{63}$

Vor diesem wissenshistorischen Hintergrund schrieb der Psychoanalytiker Sigmund Freud 1931 über das „Unbehagen der Kultur“ (Originaltitel: „Das Unglück in der Kultur“64), dass, obschon die Menschen „als Zweck“65 ihres Lebens nach einem „Glück“ strebten, „[...] die Absicht, daß der Mensch ,glücklich“ [werde] [...] im Plan der ,Schöpfung' nicht enthalten [sei]. Was man im strengsten Sinne Glück heißt, entspringt der eher plötzlichen Befriedigung hoch aufgestauter Bedürfnisse und ist seiner Natur nach nur als episodisches Phänomen möglich. “66 Vor dem Hintergrund seiner psychoanalytischen Theorie des Lustprinzips bzw.

dem es, im Ganzen seiner Existenz, alles nach Wunsch und Willen geht‘.Vgl. Eudämonismus.“ Vgl. Art. Glückseligkeit 1926. „Eudämonismus (griech. „Glückseligkeitslehre“), in der Ethik die Richtung, die das Glück im Sinne des persönlichen körperlichen und seelischen Wohlbefindens als Ziel des sittlichen Strebens bezeichnet. Eine Abart des E. ist der Utilitarismus (s.d.)“, vgl. Art. Eudämonismus 1926.

61 Art. Glück 1926.

62 Vgl. Hitzer 2011. Neurosen, Psychosen und gefühlslose Zeiten: 1880-1930. In: Frevert 2011, 125-142, 135. Zum Verhältnis zwischen Wissenschaften und Emotionen, 1880 -1930, vgl. Jensen und Morat 2008.

63 Vgl. Freiheit und Glück im Streit um den ,neuen Menschen‘. In: Thomä 2003, 33-58.

64 Vgl. Nachwort der Hrsg. In: Freud 2010, 135.

65 Freud 2010, 20. Zur Psychoanalyse und „Glück“, vgl. Vollmann 2011, 278-281.

66 Freud 2010, 21. 
der „individuellen Libidoökonomie“67 als „Methode zur Leidverhütung“68 definiert Freud „Glück“ als „das Erleben starker Lustgefühle“. ${ }^{99}$ Damit legt Freud den Fokus in erster Linie auf ein „Glück“ in der „Triebbefriedigung“70. Für den Psychoanalytiker besteht die Möglichkeit, ein „episodisches“ „Glück“, nicht aber ein „Glück“ von Dauer, zu erlangen: „Somit sind unsere Glücksmöglichkeiten schon durch unsere Konstitution beschränkt“, schlussfolgert Freud. ${ }^{71}$

In Meyers Lexikon von 1938 wird schließlich der historische Bedeutungswandel offensichtlich, den das ambivalente Glückswissen der Moderne spätestens seit dem ausgehenden 19. Jahrhundert erfahren hatte und durch die Zäsur von 1933 aufs Neue durchlief: 1. Die abschätzige Bewertung des Eudämonismus, 2. die positive Beurteilung eines ethischen Rigorismus, 3. die Verurteilung des Utilitarismus (mit dem Verweis auf Jeremy Bentham), 4. die wissenshistorische Umdeutung der Glücksbegriffe bei Kant und 5. bei Nietzsche sowie 6. die Schlussfolgerung der Lexikonautorin zur Bedeutung und Funktion von „Glück“ für eine nationalsozialistische Lebensführung.

Zum Selbstverständnis der Moderne gehört das Wissen um eine Traditionslinie innerhalb der politischen Philosophie, die auf der Ebene des Individuums mit dem Dualismus zwischen autonomer Selbstbestimmung und biologischem Selbsterhalt und auf der Ebene des Staates mit dem Antagonismus zwischen Wohlfahrtsstaat und Liberalismus um eine Lösung dieser Unvereinbarkeiten ringt. ${ }^{72}$ Im Utilitarismus liegt die Antwort auf dieses scheinbar anthropologische Dilemma im Glückswissen um eine „Bedürfnisbefriedigung“: „Unabhängig davon“, schreibt der Philosoph Thomä, „ob die Bedürfnisse auf natürliche Vorgaben oder auf eigens ausgedachte Wünsche zurückgehen, wird eine passive (das Bedürfnis verspürende) und eine aktive (das Ziel anstrebende) Seite unterschieden. ${ }^{\text {“73 }}$ Der Lexikonartikel von 1938 wirft den Utilitaristinnen ein angebliches „Aufgehen in Passivität und Gefühl“ vor. Die Kritik an der „Passivität“ der Leidenschaften, den passions, im Gegensatz zur „Aktivität“ des Geistes oder des Intellekts, wurde insbesondere durch Descartes vertreten (wie bereits erwähnt), der den Dualismus zwischen „Körper“ und „Geist“ bzw. zwischen „Gefühl“ und „Verstand“ betonte. Anstelle von Leidenschaften benannte Bentham in seinem Utilitarismus-Konzept eine Anzahl von apriorischen Bedürfnissen („hedonic cal-

67 Zu Freud und „Glück“, vgl. Thomä 2003, 54 f.

68 Freud 2010, 22.

69 Ebd., 20.

70 Ebd., 24.

71 Ebd., 21. Zur hist. Soziologie und „Glück“ vgl. Bellebaum 1997, Illouz 2009, Bosch 2012.

72 Vgl. Thomä 2003, 131-142.

73 Ebd., 144. 
culi“), an denen sich ein „Glück“ messen ließe. ${ }^{74}$ Der Lexikoneintrag von 1938 macht unmissverständlich deutlich, dass die NS-Ideologie nicht die Absicht hegte, ein individuelles Glücksbestreben zu befriedigen.

Der Lexikoneintrag zu „Staat“ im „Große[n] Brockhaus“ von 1934 zeigt, dass die Redaktion in ihrer Begriffserläuterung davon absah näher darauf einzugehen, dass es in wissenshistorischer Kontinuität zur Aufklärung zu den Staatsaufgaben gehörte, einen „freien Staatsbürger“ zur autonomen Selbstbestimmung im Sinne des moralisch-tugendhaften Vernunftprinzips nach Kant zu erziehen: „Er [der Staat] erzieht nicht mehr zum ,freien Staatsbürger', sondern kennt nur eine Erziehung zu Volk und Staat und stellt die Jugend bewußt in die Bindungen an Volk und Staat.“75 Hatte die 15. Auflage ${ }^{76}$ je einen neuen Eintrag zum „Bürgertum“ (Bd. 3, 1929) $)^{77}$ und zur „Gesellschaft“ $(1 \mathrm{Bd} .7,1930)^{78}$ enthalten, in welchen auf verschiedene Gesellschafts- und Staatstheorien (Marxismus, Liberalismus, Universalismus etc.) hingewiesen und im Sinne der Soziologie (einer damals jungen Wissenschaftsdisziplin) auf soziale Gesellschaftsbedingungen eingegangen wurde, legte der 1934 erschienene Artikel zu „Staat“ eine eindeutig normative Wertung nahe: Die faschistische und nationalsozialistische Diktatur wurde unmissverständlich dem Liberalismus und der Demokratie gegenübergestellt. Der Nationalsozialismus zog eine „Erziehung zu Volk und Staat“ im Konzept eines „deutschen“ „Volkskörpers“ vor.

Daran anknüpfend zeigt der Vergleich der Kant-Rezeptionen in den Ausgaben von Meyers Lexikon von 1926 und 1938, wie sich der innert rund 12 Jahren vollzogene Bedeutungswandel von Glückswissen manifestieren konnte. In Meyers Lexikoneintrag zur „Glückseligkeit“ von 1926 heißt es:

74 Bentham: „An Introduction to the Principles of Morals and Legislation“, [1780/1789]. In: John Stuart Mill. 1979. Utilitarism. On Liberty. Essay on Bentham. New York: Scarborough, 33-77, zit. nach Thomä 2003, 147. Benthams Theorie übte auf die britischen Sozialreformer des frühen 19. Jahrhunderts großen Einfluss aus, die in der Sicherstellung der gesellschaftlichen Bedürfnisbefriedigung ein zentrales Ziel sahen. In Romanen wie Jewgeni Samjatins „Wir“ (1924), Aldous Huxleys „Brave New World“ (1932) oder George Orwells „1984“ (1949) wurde das staatlich verordnete Glücksversprechen kritisch thematisiert. Ein prominentes Beispiel des U. ist die Formel des pursuit of happiness in der Amerikanischen Unabhängigkeitserklärung (1776). Aktuelle ökonomische Glücksforscher wie Richard Layard (Happiness-Index) verhelfen dem U. seit den 1990er Jahren zu neuer wirtschaftspolitischer Bedeutung.

75 Art. Staat 1934. Für den Passagenhinweis vgl. Haltern 1976, 90.

76 Die neuaufgelegten Bände des Großen Brockhaus erschienen als 15. Auflage zwischen 1928 und 1935, also gegen Ende der Weimarer Republik (1918-1933) und zu Beginn der NS-Zeit. Vgl. Haltern 1976, 90.

77 Art. Bürgertum 1929.

78 Art. Gesellschaft 1930. 
„Glückseligkeit (griech. Eudämonie), der Zustand des sinnlich-vernünftigen Wesens, in dem es nicht nur seine Bedürfnisse, sondern auch seine Wünsche befriedigt sieht, oder (nach Kant) ,dem es, im Ganzen seiner Existenz, alles nach Wunsch und Willen geht‘. Vgl. Eudämonismus." ${ }^{79}$

Die lexikalische NS-Rezeption Kants von 1938, wonach der Philosoph „für die Pflicht gegen das Glück“ kämpfe, greift, mit Blick auf das obige Zitat (,dem es, im Ganzen seiner Existenz, alles nach Wunsch und Willen geht“), zu kurz. Kant lehnte zwar einen teleologischen Eudämonismus ab, jedoch nicht das tugendhafte Handeln als eine Quelle von „Glück“ über dessen Gelingen. ${ }^{80}$ Dennoch plädiert der Lexikoneintrag von 1938, mit Bezug auf Kant (und Nietzsche), ausschließlich für Aktivität statt einer Passivität, für das Handeln statt des Fühlens und betont dabei die Wichtigkeit der Erschaffung eines Werks.

Doch auch hier muss differenziert werden. Nietzsche wurde aus unterschiedlichen Gründen für die nationalsozialistische Ideologie instrumentalisiert: Zunächst galt Nietzsche als einer der schärfsten Kritiker des Utilitarismus: „Der Mensch strebt nicht nach dem Glück; nur der Engländer thut das“81, lautet Nietzsches berühmt gewordener Ausspruch, der eindeutig auf den britischen Utilitarismus abzielte. Die Utilitarist`innen bezeichnet Nietzsche als eine „mittelmässige Art Mensch“ und die „Wohlfahrt“ als „Brechmittel“. ${ }^{82}$ Nietzsche umschreibt in „Also sprach Zarathustra“ (von wo der Lexikonartikel von 1938 das Zitat „Trachte ich [...]“ entnommen hat) all jene Menschen, die nach „Glück“ strebten, als „letzte Menschen“ und rühmt im Gegensatz dazu den „Übermenschen“. ${ }^{83}$ Die wissenshistorische Kontinuität der NS-,,Rassenlehre“ mit seiner Vorstellung von einem hegemonialen, „kämpferischen“ Ariertypus (dem „nordischen Mensch[en]“, wie es im Artikel heißt) wird in der Nietzsche-Rezeption des Lexikonartikels von 1938 deutlich. Doch wie begründete Nietzsche seine Haltung?

„Ob Hedonismus [...] ob Utilitarismus, ob Eudämonismus: alle diese Denkweisen, welche nach Lust und Leid, das heisst nach Begleitzuständen und Nebensachen den Werth der Dinge messen, das sind [...] Naivitäten, auf welche ein Jeder, der sich gestaltender Kräfte und eines Künstler-Gewissens bewusst ist, nicht ohne Spott (...) herabblicken wird. [...] Wohlbefinden, wie ihr es versteht - das ist ja kein Ziel, das scheint uns ein Ende! ${ }^{* 84}$

79 Eudämonismus Art. Glückseligkeit 1926

80 Zum moralischen Glücksbegriff in der Kantischen Ethik vgl. Mitscherlich-Schönherr 2011.

81 Nietzsche 1988, 61. Für den Passagenhinweis vgl. Thomä 2003, 169.

82 Nietzsche, Bd. 5, $164 \mathrm{f}$

83 Ebd., Bd. 4, $19 \mathrm{f}$.

84 Ebd., Bd. 5, $160 \mathrm{f}$. 
Wissenshistorisch betrachtet dient das Nietzsche-Zitat in Meyers Lexikon zur Legitimation eines Handelns, bei welchem (individuelles) „Glück“ folglich kein Lebensziel darstelle. ${ }^{85}$

Mit den Ausdifferenzierungen der Wissenschaftsbereiche im Verlauf des 19. Jahrhunderts, die mit dem Anspruch auf wissenschaftliche Objektivierung einhergingen, waren beispielsweise die Herausgeber`innen des „Großen Brockhaus“ darauf bedacht, normative Wertungen in den Lexikonartikeln längerfristig zu vermeiden. Stattdessen sollte sich die Autorenschaft an den aktuellen, wissenschaftlichen Publikationen des jeweiligen Themengebiets orientieren. Diese Errungenschaft fand mit der Machtergreifung der Nationalsozialisten im Frühjahr 1933 ein jähes Ende. Die Ideologie der NS-Diktatur schlug sich offenkundig in der Deutungshoheit der Lexikoneinträge nieder. ${ }^{86}$ Die Untersuchung hat aufgezeigt, wie sich der Wandel lexikalischen Glückswissens im wissenshistorischen Vergleich unterschiedlicher Editionen untersuchen lässt. Darüber hinaus hat die Untersuchung die wissenshistorische Beziehung zwischen lexikalischem Glückswissen und der NS-Moral „Kraft durch Freude“ exemplarisch beleuchtet. An diese wissenshistorische Erkenntnisse knüpft das nächste Kapitel an.

\section{4 „Glück“ oder „Pflicht“? NS-Arbeitsmoral in der nationalsozialistischen Lebensführung}

Der Lebensentwurf eines „arischen“ „Volksgenossen“ sollte der „Lebensführung des nationalsozialistischen Deutschland [sic]“ entsprechen, schrieb Prof. Dr. Walter Groß (1904-1945), Leiter des „Rassenpolitischen Amtes der NSDAP“ in seinem Aufsatz zur „Nationalsozialistische[n] Lebensführung“. ${ }^{87}$ Die totalitäre „politische Neuordnung seines Lebensraumes“ sah vor, nicht nur die „Haltung und Lebensführung des Volkes“, sondern auch jene „seiner einzelnen Glieder“ zu erfassen. $^{88}$ Die individuelle Lebensführung jedes „Einzelnen“89 und das Bewusstsein eines psychologischen „Ich[s]“ sollte nicht länger nach einem individuellen Streben nach „Glück“ gestaltet werden, wie es zahlreiche Glücksratgeber ihrer Leserschaft seit der Jahrhundertwende rieten. ${ }^{90}$

85 Die aktuelle Nietzsche-Rezeption betont Nietzsches ambivalente Haltung zu „Glück“, vgl. Thomä 2003, 182. Zur Nietzsche-Rezeption in exemplarischen Ratgebern s. Kap. 10.1.

86 Vgl. Haltern 1976, 62.

87 Vgl. Groß 1942, 59. Mehr zu Groß vgl. u. a. Uhle 1999, Uhle 2017.

88 Vgl. Groß 1942, 59.

89 Ebd., 59.

90 Vgl. Kleiner und Suter 2015. 
Mit Blick auf den II. Hauptteil lautet, ausgehend von Groß’ Definition einer „nationalsozialistischen Lebensführung“, eine zentrale These der vorliegenden Untersuchung: Die nationalsozialistischen Ratgeberautor`innen leiteten ihre Leserschaft $\mathrm{zu}$ einer leistungsorientierten Lebensführung im Rahmen einer affirmativen Gefühlspolitik an, die eine „Entfaltung und möglichst vollkommene Ausbildung der Persönlichkeit“ im Sinne der „rassenhygienischen“ Moralvorstellung vorsah. ${ }^{91}$ Diese scheinbar individualisierte, aber dennoch totalitäre Persönlichkeitskultur hatte zum Ziel, das „nationalsozialistische Leben“ als „Dienst des Einzelnen an der Gemeinschaft“ $z u$ entwerfen. ${ }^{92}$ Aus diesem teleologischen Lebenskonzept sollte jede „Volkgenossin“ „eigene[...] Kräfte“ entfalten und „Bewußtsein“ sowie „Freude“ schöpfen, als „Voraussetzung des vollen Einsatzes und höchsten Wertes für die Gemeinschaft des Volkes“.93

Groß definierte die wesentlichen Merkmale der „nationalsozialistischen Lebensführung“ im Sinne einer leistungsorientierten, „rassenhygienischen“ Gefühlskultur aus moralischer Überzeugung und emotionspolitischer Empfindung in Ambivalenz zwischen „Glück und Pflicht“:

„Wie die Weltanschauung des Nationalsozialismus, so ruht auch ihre praktische Verwirklichung in der Lebensführung der deutschen Menschen auf zwei tragenden Säulen: Die Entfaltung und möglichst vollkommene Ausbildung der Persönlichkeit und der Dienst des Einzelnen an der Gemeinschaft sind die beiden Pole, zwischen denen die fruchtbare Spannung nationalsozialistischen Lebens in Deutschland entsteht. [...] Umgekehrt aber verleiht erst dieser Dienst am Ganzen dem eigenen Ich und seiner Entwicklung den letzten Adel und den tiefsten Sinn. Persönlichkeit und Gemeinschaft, in einer kranken Zeit zu Gegensätzen entwickelt, sind für uns ein voller, tönender Akkord, in dem die Einzelsaiten unseres Lebens zusammenklingen. [...] Niemals aber kann von echtem Nationalsozialismus in Haltung und Gestaltung des Lebens die Rede sein, wo nicht der Wunsch nach der eigenen Familie und der großen Zahl eigener Kinder und das Bewußtsein von Glück und Pflicht zugleich vorhanden sind, die im Kinderreichtum des Volkes liegen.“94

Das individuelle Glücksstreben erreichte aus Sicht der NS-Ideologen seinen Höhepunkt in der als „Krise“95 gedeuteten Weimarer Republik. Um dem entgegenzuwirken, sollte der „Volksgenosse“ als „neuer Mensch“ in die „arische Volksgemeinschaft" ${ }^{“ 96}$ eingebunden werden. Vordergründig würde er erst in diesem

91 Vgl. Groß 1942, 59.

92 Vgl. ebd.

93 Vgl. ebd.

94 Ebd.

95 Zum Krisendiskurs in der Weimarer Republik vgl. Eitz 2015, Eitz und Engelhardt 2015. Zum Krisendiskurs im Kontext der Ratgeberliteratur vgl. Peeters 2021.

96 Zur „Volksgemeinschaft“ vgl. Wildt 2007a, Reinicke u. a. 2014, Steber und Gotto 2014. 
Verbund die wahre „Freiheit“ erlangen und sein „Glück“ finden, in dem er im Sinne der NS-Arbeitsideologie „Kraft durch Freude“ erlange.

Hintergründig bezweckten die explizit emotionspolitisch formulierten Propagandaabsichten die Stabilisierung des neuen Regimes. ${ }^{97}$ Zahlreiche über den NSJahreskalender verteilte Kulturveranstaltungen (s. Kap. 13, 15, 16) wurden zur Veranschaulichung der vermeintlich realisierten Politik einer „rassenideologischen" Gefühlskultur instrumentalisiert. Organisiert wurden diese Anlässe vielerorts von der Freizeitorganisation „Kraft durch Freude“ und waren häufig von Feierlichkeiten wie Jubiläen, Preisverleihungen und Ehrungen umrahmt. „Mit kulturellen Veranstaltungen wollten sie [die Nazis] die Lebensfreude der Menschen erhöhen und sie zudem glauben machen, dass sich die Wirklichkeit verändere“, so der Historiker Peter Reichel. ${ }^{98}$

„NS-Rassenideologen“ wie Walter Groß betonten dabei die „Vielfältigkeit und Unterschiedlichkeit der Einzelmenschen“ im erwachten „Bewußtsein“ über das „unerbitterliche Gesetz der Erblichkeit“. Groß bewarb eine scheinbar individuell ausgerichtete Entfaltungsmöglichkeit der „Persönlichkeiten“ jeder/s „Einzelnen“. Dabei versuchte er seinen „rassenhygienischen“ Utopiegedanken eines heranzuzüchtenden „reinrassigen“ „Volkskörpers“, dem ein „genormter Massentypus Mensch“ und die charakterologische Normierung „völkischer“ „Anlagen und Fähigkeiten in der Gemeinschaft des Volkes“ zugrunde lag, propagandistisch zu verschleiern:

„Darum kann nationalsozialistische Lebensführung nicht dem falschen Trugbild oder Gleichmacherei und verbindlicher Normen für Alle dienen. Nicht ein langweiliges Modeideal, nicht ein genormter Massentypus Mensch ist ihr Ziel, sondern die Mannigfaltigkeit eigenartiger, nach ihrer jeweiligen Anlage entwickelter und ausgebildeter Persönlichkeiten, deren Vielfalt und gegenseitige Ergänzung den Einzelnen erhöht, dem Volke aber erst seine geschichtliche Kraft und Schönheit verleiht.“99

In Kapitel 8 wird weiterführend darauf Bezug genommen, inwiefern lexikalisches Glückswissen für den Entwurf einer nationalsozialistischen Lebensführung als eine affirmative Gefühlskultur einer wissenshistorisch ambivalenten Dichotomie aus ,Gefühl‘ und ,Verstand‘ Anwendung fand.

97 Zur Weimarer Republik um 1930 vgl. Ehrlich und John 1998a. Zu (Dis-)Kontinuitäten im Nationalsozialismus vgl. Kundrus und Steinbacher 2013.

98 Reichel 2006, 310.

99 Groß 1942, 60. 